\title{
Electrons, photons and muons in ATLAS
}

\author{
Alexandre Solodkov \\ for the ATLAS Collaboration \\ Institute for High Energy Physics, RU-142280, Protvino, Russia \\ e-mail: solodkov@mx.ihep.su
}

\begin{abstract}
ATLAS is a general purpose experiment which will operate at the LHC. In this paper, the detector performance in terms of electrons, photons and muons identification and measurements are presented, combining full GEANT simulation and test beam results. Some of the prominent methods and event samples which will be used in situ to perform the calibration and evaluate the absolute energy scale are described as well. It is finally shown how these performances will allow ATLAS to accurately reconstruct signal mass peaks in several physics benchmark channels.
\end{abstract}

PACS: 25.70.Ef; 21.60.Gx; 27.30.+t

\section{Introduction}

The ATLAS experiment - which has now fully entered the construction phase - will operate at the LHC proton-proton collider at a luminosity of $10^{34} \mathrm{~cm}^{-2}$ $s^{-1}$. The goal of the ATLAS detector is to be sensitive to as many signatures as possible in order to achieve a robust and redundant physics measurement in such an harsh environment. In the following, the capability of the single ATLAS subsystems as well as of their combination in identifying and measuring electrons, photons and muons will be discussed. The basic detector design criteria which are of interest here, include:

- a very good electromagnetic calorimetry for electron and photon identification and measurements,

- very high-precision muon momentum measurements, with the capability to guarantee accurate measurements at the highest luminosity using the external spectrometer alone,

- an efficient tracking at high luminosity for high $p_{T}$ lepton measurements and electron and photon identification

- a large acceptance in pseudorapidity $(\eta)$ with almost full azimuthal angle $(\phi)$ coverage everywhere.

\section{Standalone Inner Detector performance}

\subsection{Inner Detector Layout}

The layout of the ATLAS Inner Detector (ID) is shown in Fig.1 (more details can be found in [1],[2]). Pattern recognition, momentum and vertex measurements 


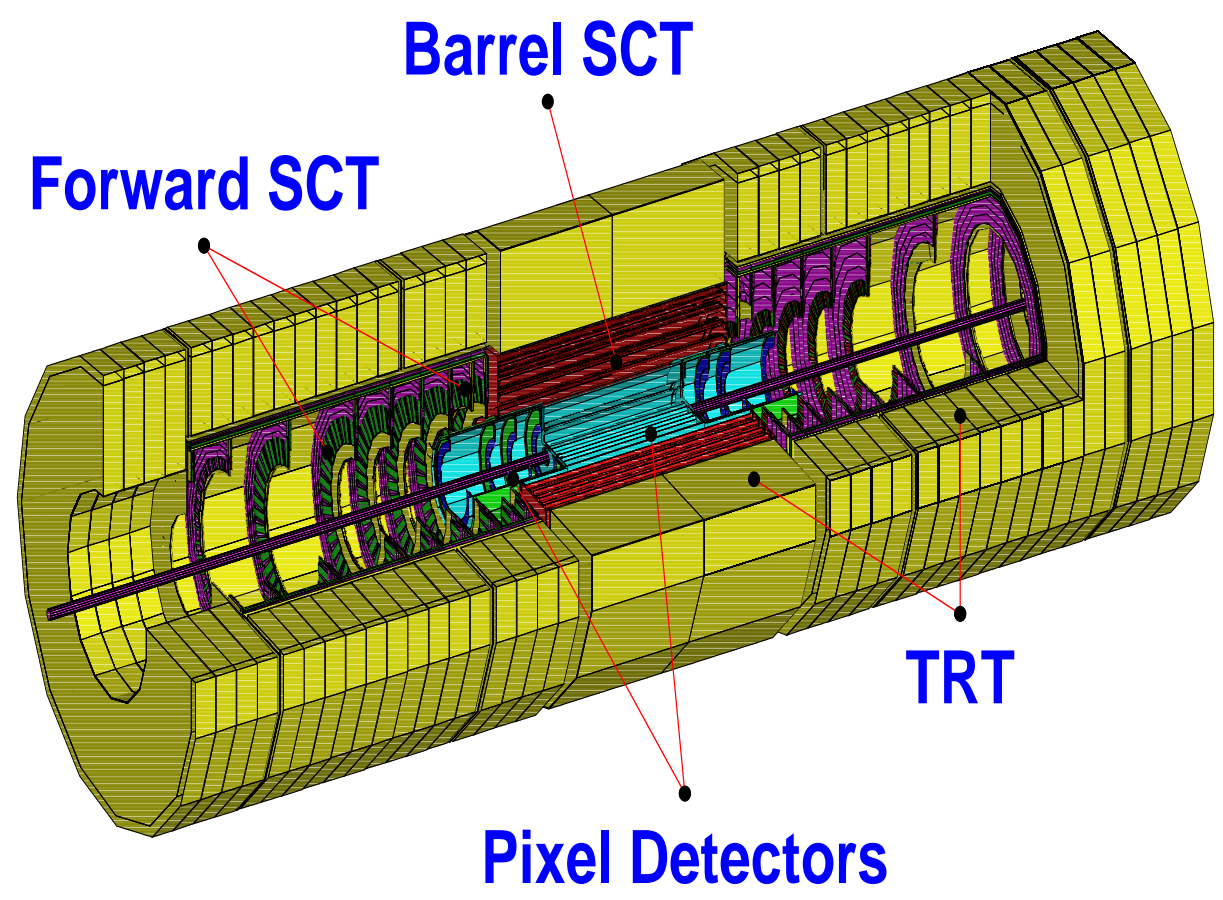

Fig. 1. Three-dimensional view of the ATLAS Inner Detector.

and electron identification are achieved by combining the information from three different systems.

The Pixel detector is designed to provide a very high-granularity, high precision set of measurements as close to the interaction point as possible. The system provides three precision measurements over the the full acceptance, and mostly determines the impact parameter resolution and the ability of the Inner Detector to find short-lived particles.

The Semiconductor Tracker (SCT) gives eight precision measurements (four space points) per track in the intermediate radial range, contributing to the measurement of momentum, impact parameter and vertex position. In addition, it provides a good pattern recognition thanks to its high granularity.

The Transition Radiation Tracker (TRT) is based on the use of straw detectors, which can operate at the very high rates. Electron identification capability is added by employing Xenon gas to detect transition-radiation photons created in a radiator between the straws. Typically, about 36 measurements per track can be obtained.

\subsection{Momentum resolution}

The amount of material in the inner cavity has an impact on the performance of the ID and of the electromagnetic (EM) Calorimeter. The material budget for the present ID layout is shown in Fig.2. 


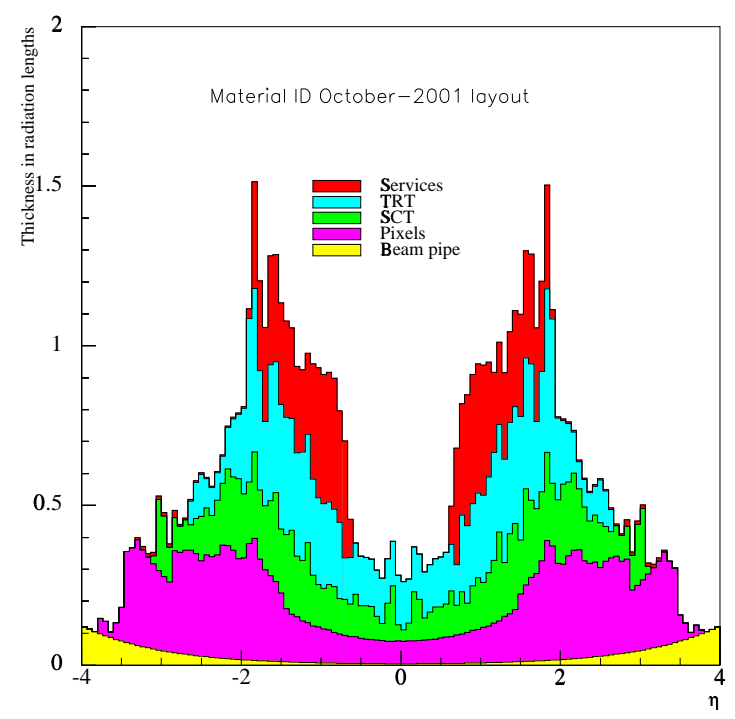

Fig. 2. Material distribution of the ID vs $\eta$ for the 2001 layout. The various bands include all services and support structures within the corresponding fiducial volumes. The services outside the TRT are shown separately.

The resolution in the transverse impact parameter $\left(d_{0}\right)$ and in $1 / p_{T}$ ( where $p_{T}$ is the transverse momentum) for the muon-like tracks are given by the formulae:

$$
\begin{gathered}
\sigma\left(d_{0}\right) \approx 11 \oplus 98 /\left(p_{T} \sqrt{\sin \Theta}\right) \\
\sigma\left(1 / p_{T}\right) \approx 3.6 \times 10^{-4} \oplus 1.3 \times 10^{-2} /\left(p_{T} \sqrt{\sin \Theta}\right)
\end{gathered}
$$

These formulae apply to the tracks in the range from $\mathrm{GeV}$ to $\mathrm{TeV}$. For $p_{T}=$ $20 \mathrm{GeV}(100 \mathrm{GeV})$ it corresponds to $\sigma\left(p_{T}\right) / p_{T}=1.5 \%(3.8 \%)$

Fig.3 and Fig.4 compare the reconstructed $1 / p_{T}$ and impact parameter distributions for $20 \mathrm{GeV}$ muons, pions and electrons generated in the full $\eta$ range $|\eta|<2.5$. Since pions have a low probability to interact within the ID, their distributions are similar to those of muons. Electrons are affected by bremsstrahlung and this produces tails, which are around $30-50 \%$ in the $1 / p_{T}$ distribution and $10-15 \%$ in the case of the impact parameter distribution. Similar effects are seen at $p_{T}=1 \mathrm{GeV}$, although the electron tails are reduced by about a third.

\subsection{Electron momentum measurements}

Since $20 \%$ of electrons lose half of their energy after traversing the ID (mostly due to bremsstrahlung), the measurement of the initial electron momentum is a difficult task. Most of the bremsstrahlung energy is collected by the EM Calorimeter, but radiation changes track curvatures and reduces the reconstructed $p_{T}$. However, it is possible to correct for this effect, improving the $p_{T}$ measurement and 


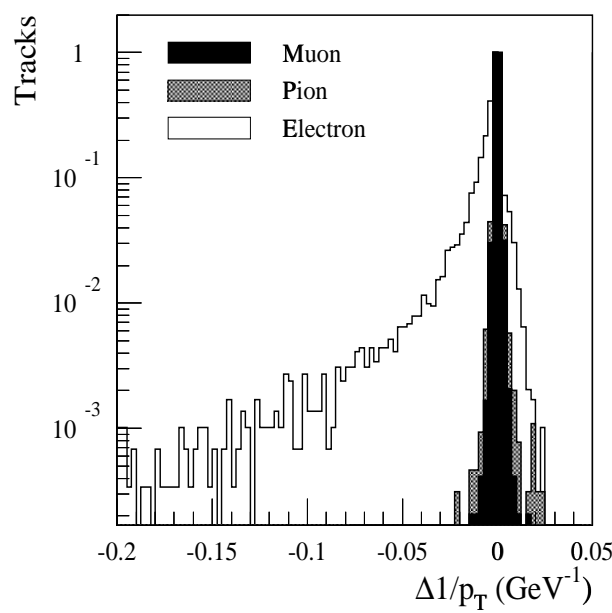

Fig. 3. Difference between the reconstructed and generated $1 / p_{T}$ for single $20 \mathrm{GeV}$ negatively charged particle.

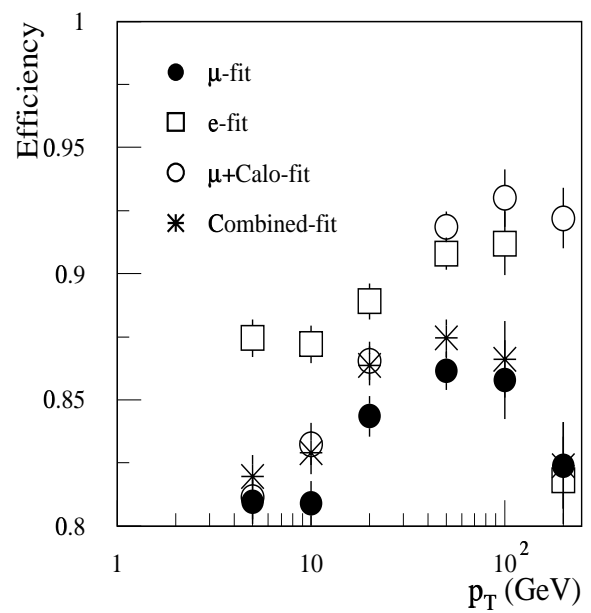

Fig. 5. Efficiency for reconstructing electrons $(\eta=0.3)$ using different fits. Electrons are requested to have a "good" track in the ID and $0.7<$ $p_{T}^{g e n} / p_{T}<1.4$.

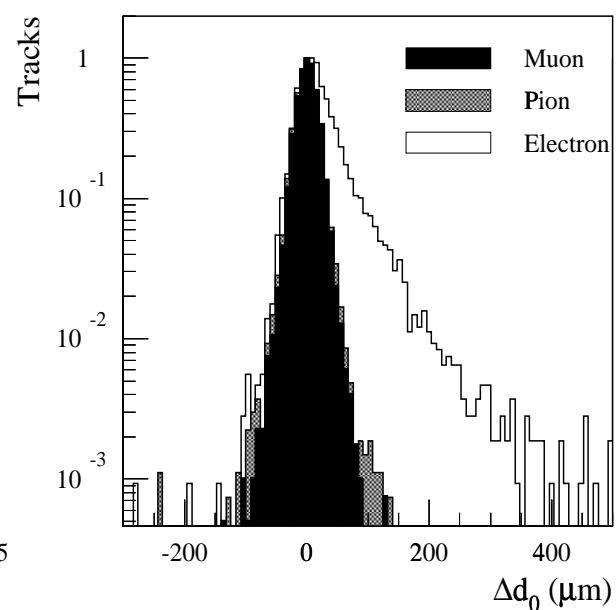

Fig. 4. Difference between the reconstructed and generated transverse impact parameter for single $20 \mathrm{GeV}$ negatively charged particle.

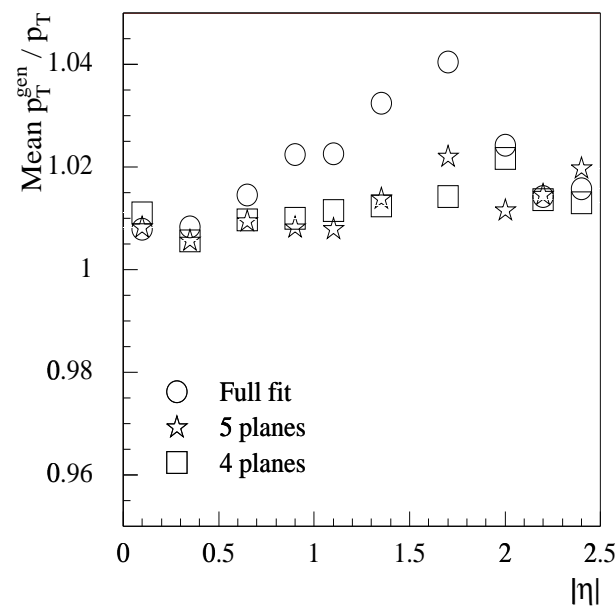

Fig. 6. Ratio of true $p_{T}$ to reconstructed $p_{T}$ for $p_{T}=20 \mathrm{GeV}$ electrons as a function of $\eta$. Track fits are made to either the full track or to a reduced number of silicon planes.

increasing the acceptance. To perform these corrections different approaches can be used. In Fig.5 and Fig.6 two examples of possible methods of correction are shown. On the left plot the efficiency for reconstructing electrons at $\eta=0.3$ is shown. The "muon fit" is the the fit which is appropriate for muons, but 
makes no allowance for electron bremsstrahlung. The "electron fit" does allow for radiation and the efficiency is increased by $\sim 6 \%$, although it degrades the $p_{T}$ resolution by a factor of 2 . The "combined fit" increases the efficiency by $\sim 2 \%$, while retaining good resolution. The use of the EM cluster position in the "muon+Calo fit" significantly improves the tails and hence the efficiency. The right plot shows the ratio of the true $p_{T}$ to the reconstructed $p_{T}$ for $20 \mathrm{GeV}$ electrons as a function of $\eta$. Track fits are made to either the full track or to a reduced number of silicon planes. It can be seen that as the track is truncated, the mean gets closer to one, indicating that there is less sensitivity to bremsstrahlung. However, the resolution is degraded.

\subsection{Charge determination}

Once a track is reconstructed, the particle charge can be determined as well. This information is important, for example, in the case of the investigation of charge asymmetries in $Z^{\prime}$ decays. It should be noted that a good $p_{T}$ resolution is required at high $p_{T}$ to reduce the error in the charge determination. In Fig.7 the fraction of wrong sign assignments is given as a function of the track $p_{T}$ for the cases of muons and electrons in the presence of a solenoidal or a uniform magnetic field. The solenoidal magnetic field, which is expected in the ATLAS inner cavity, and the ideal uniform magnetic field were studied to see the effect of nonuniformity in the B-field. For muons, the behaviour of the curve is well described by the intrinsic resolution. Reconstruction of the electron tracks is complicated by the bremsstrahlung. Nevertheless, at $500 \mathrm{GeV}$ the sign of the electron can be determined to better than three standard deviations, which corresponds to a misidentification probability of $0.13 \%$. The electron charge reconstruction is satisfactory up to $\approx 1 \mathrm{TeV}$.

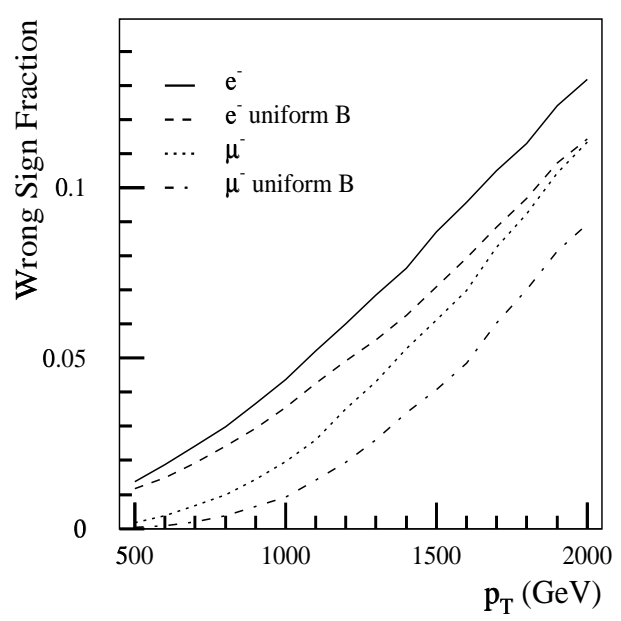

Fig. 7. Wrong sign fraction as a function of $p_{T}$ for muons and electrons, averaged over $\eta$ in the presence of either a solenoidal or a uniform magnetic field. 


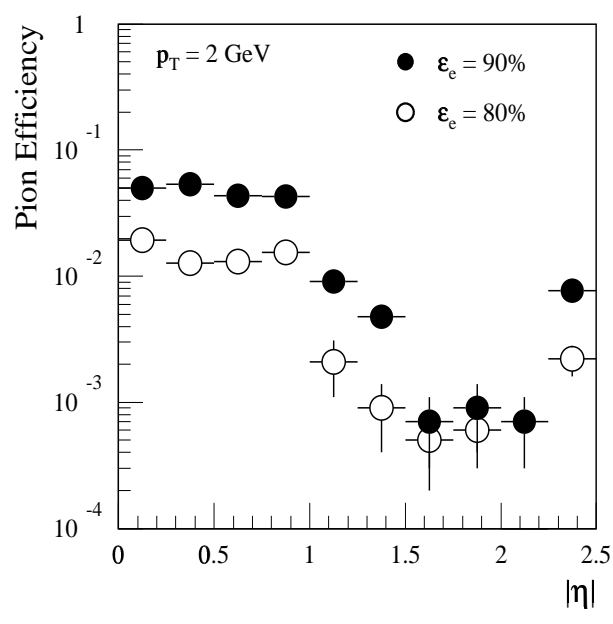

Fig. 8. Pion efficiency as a function of $|\eta|$ for $p_{T}=2 \mathrm{GeV}$ for two different electron efficiencies.

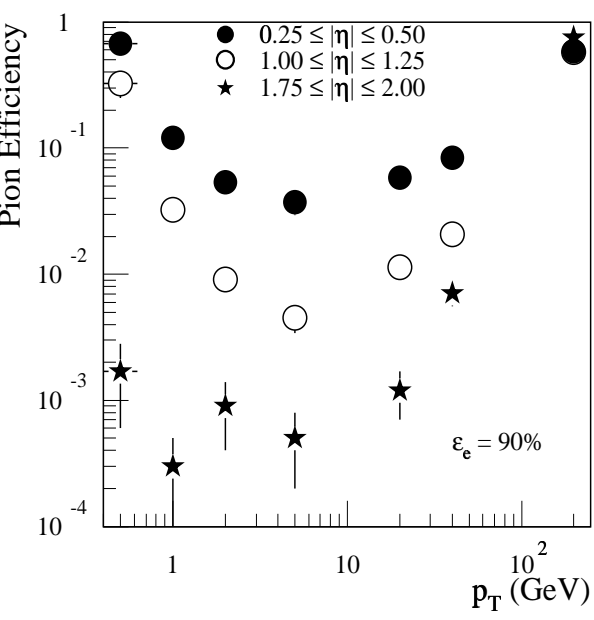

Fig. 9. Pion efficiency as a function of $p_{T}$ for various $\eta$ intervals for a $90 \%$ electron efficiency.

\subsection{Particle identification in the TRT}

The transition radiation measurements in the TRT provide separation between hadrons and electrons. Rejection against charged pions is achieved by counting the fraction of TRT straws which have a high threshold hit. Fig.8 shows the probability to misidentify a pion as a function of $\eta$, assuming an electron efficiency of $90 \%$ (upper curve) or $80 \%$. A rejection factor of more than 1000 can be achieved in the end-cap region of the TRT. Fig.9 shows how the pion efficiency varies as a function of $p_{T}$ for three different $\eta$ regions for a $90 \%$ electron efficiency. At $p_{T}>200 \mathrm{GeV}$ pions and electrons emit transition radiation photons at a compatible rate, and therefore they cannot be separated.

In Fig. 10 the beam test results of pion versus electron efficiency are shown for $20 \mathrm{GeV}$ particles. The results are in good agreement with simulation.

\section{Standalone calorimeter performance}

A view of the ATLAS calorimeters [3] is presented in Fig.11. The calorimetry consists of the EM Calorimeter covering the pseudorapidity region $|\eta|<3.2$, the Hadronic Barrel Calorimeter covering $|\eta|<1.7$, the Hadronic End-cap Calorimeter covering $1.5<|\eta|<3.2$, and the Forward Calorimeter covering $3.1<|\eta|<4.9$.

The Hadronic Barrel Calorimeter is based on a sampling technique with plastic scintillator plates (tiles) embedded in an iron absorber. All other calorimeters are liquid-argon (LAr) calorimeters with lead, copper and tungsten absorber in the EM, hadronic end-cap and forward part respectively. 


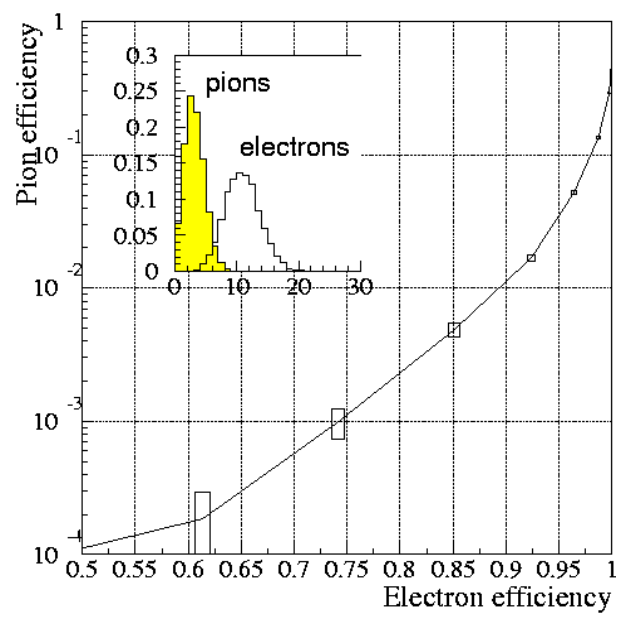

Fig. 10. TRT beam test results for the efficiency of pion vs electron identification at $20 \mathrm{GeV}$.

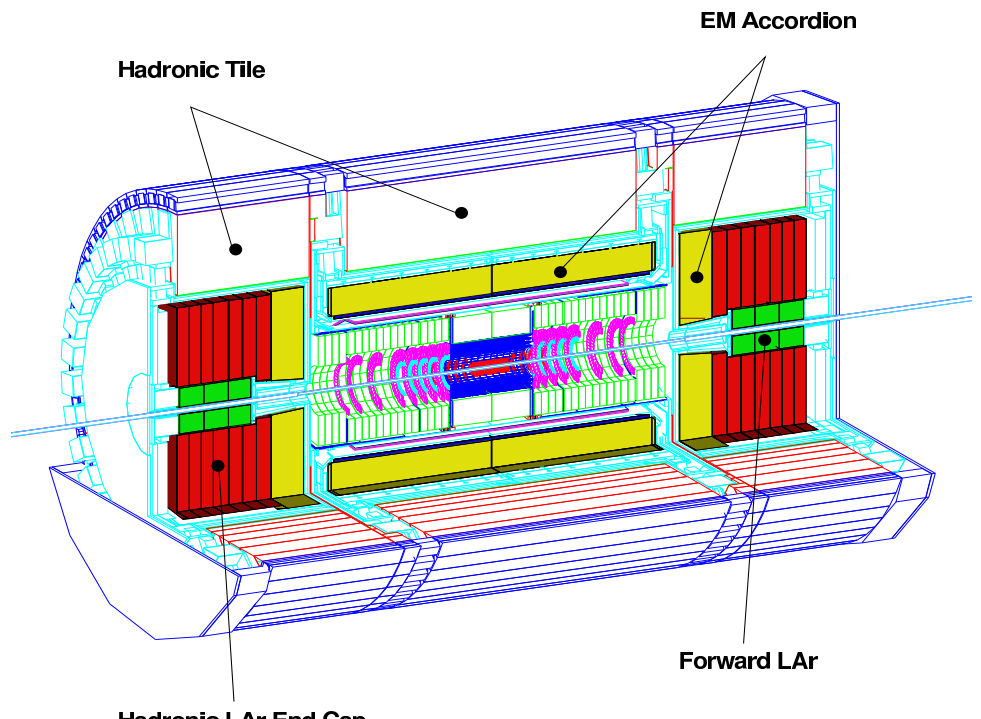

Hadronic LAr End Cap

Fig. 11. Three-dimensional view of the ATLAS calorimeters.

\subsection{EM Calorimeter layout}

The EM Barrel Calorimeter [4], shown in Fig.12, has an accordion geometry. It is divided in three longitudinal samplings characterised by a high granularity. A presampler detector is placed in front of the calorimeter to correct for the energy losses in the material upstream of the calorimeter.

The first longitudinal sampling is equipped with $4 \mathrm{~mm}$ strips in the $\eta$ direction. This section acts as a "preshower" detector, enhancing particle identifica- 


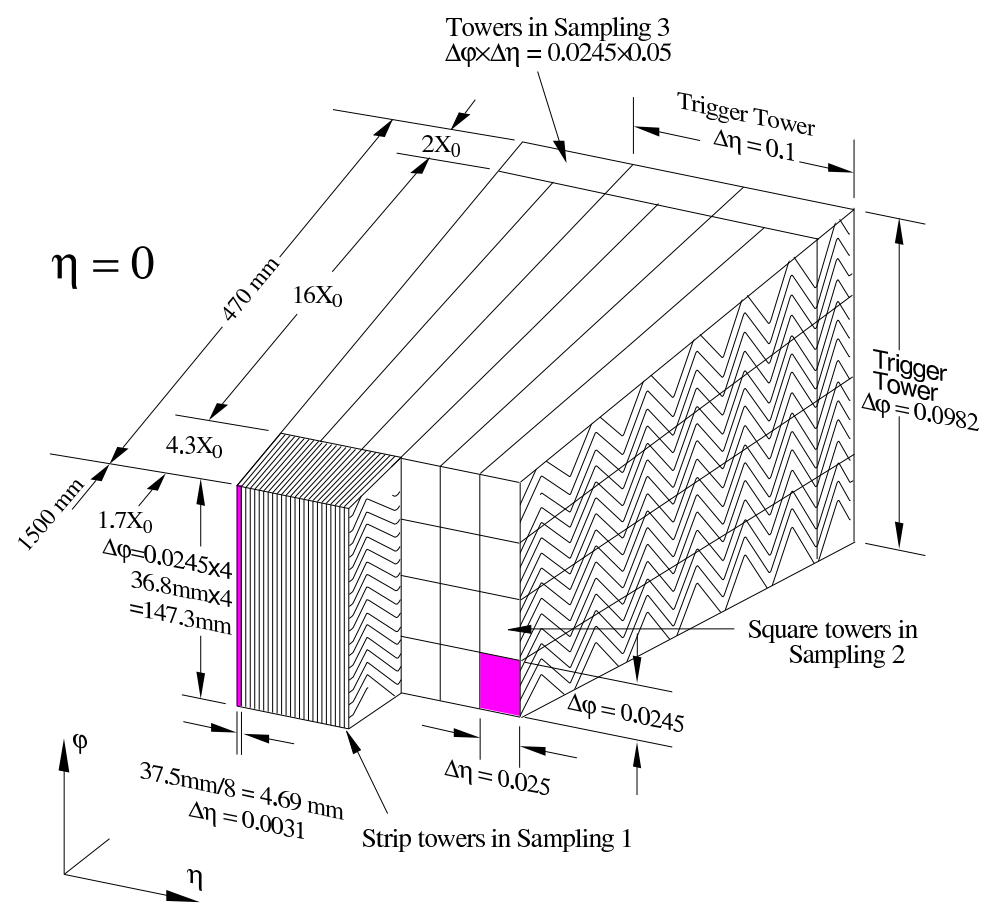

Fig. 12. Sketch of the accordion structure of the EM Calorimeter.

tion $\left(\gamma / \pi^{0}, e / \pi\right.$, etc. $)$ and providing a precise position measurement in $\eta$.

All the parameters of the EM Calorimeter are summarised in Table 1.

\begin{tabular}{r|c|ll} 
sampling & $\Delta L(\eta=0)$ & $\Delta \eta \times \Delta \phi$ & $\sigma(E) / E \approx 10 \% / \sqrt{E} \oplus 0.7 \%$ \\
\cline { 1 - 3 } presampler & & $0.025 \times 0.1$ & \\
$1^{\text {st }}$ samp & $6 X_{0}$ & $0.003 \times 0.1$ & $\sigma(R \phi) \approx 9 \mathrm{~mm} / \sqrt{E}$ \\
$2^{\text {nd }}$ samp & $18 X_{0}$ & $0.025 \times 0.025$ & \\
$3^{\text {rd }}$ samp & $6 X_{0}$ & $0.050 \times 0.025$ & $\sigma(R \eta) \approx 3 \mathrm{~mm} / \sqrt{E}$
\end{tabular}

Table 1. Parameters of the EM Barrel Calorimeter.

\subsection{Energy measurements}

The energy in the EM Calorimeter is reconstructed by summing up the energies in clusters of cells in the three longitudinal samplings, and the weighted presampler energy. The presampler weight is adjusted to recover energy loss in the upstream material. The energy resolution can be expressed as follows, where $a$, $b$ and $c$ are the sampling, noise and constant terms.

$$
\sigma(E) / E=a / \sqrt{E} \oplus b / E \oplus c
$$

The goal for the ATLAS calorimetry is to achieve a global constant term of less then $0.7 \%$, which requires a local constant term (for a single module in the beam 
test) to be less then $0.5 \%$.

Preliminary results of the beam test performed in the year 2000 on a preproduction EM Barrel Calorimeter module (module zero) are shown in Fig.13. A sampling term of less then $10 \%$ and a local constant term of about $0.3 \%$ have been achieved.

Full simulation of the calorimeter including electronic noise and pile-up shows that the resolution for $10 \mathrm{GeV}$ electrons is around $5 \%$.

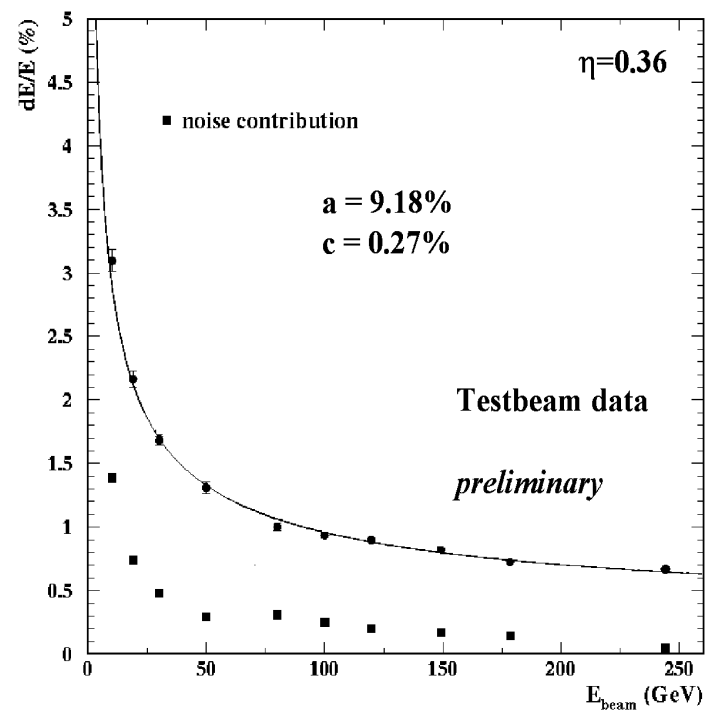

Fig. 13. Energy resolution vs beam energy for the EM Barrel Calorimeter module zero (beam test results). " $a$ " and " $c$ " are the sampling and constant terms. The noise contribution $(b / E$ term) is shown separately.

\subsection{Position and angular resolution}

The excellent longitudinal and lateral granularity of the EM Calorimeter, in particular of the strip section, allows high precision measurements of the shower position. The $\phi$ position can be measured in the second sampling and the $\eta$ position in the strip section and in the second sampling. As it is shown in Fig.14, using strips of the first sampling, a $400 \mu \mathrm{m}$ precision can be achieved. The resolution in the second sampling is of about $1 \mathrm{~mm}$. By combining the two $\eta$ measurements together it is possible to determine the shower direction in $\theta$ with an accuracy of $\sigma \approx 60 \mathrm{mrad} / \sqrt{E}$. In $H \rightarrow \gamma \gamma$ events, two photons are available in the final state, and the primary vertex can be reconstructed as the weighted average of the two vertices provided by the photons, plus the constraint that the nominal vertex position is at $z=0$ and has a dispersion of $\sigma=5.6 \mathrm{~cm}$. The vertex obtained in this way is compared to the true vertex in Fig.15. The precision of the vertex measurement provided by the calorimeter alone, averaged over the full $\eta$ coverage is $1.3 \mathrm{~cm}$.

http://link.springer.de/link/service/journals/10105/index.html 


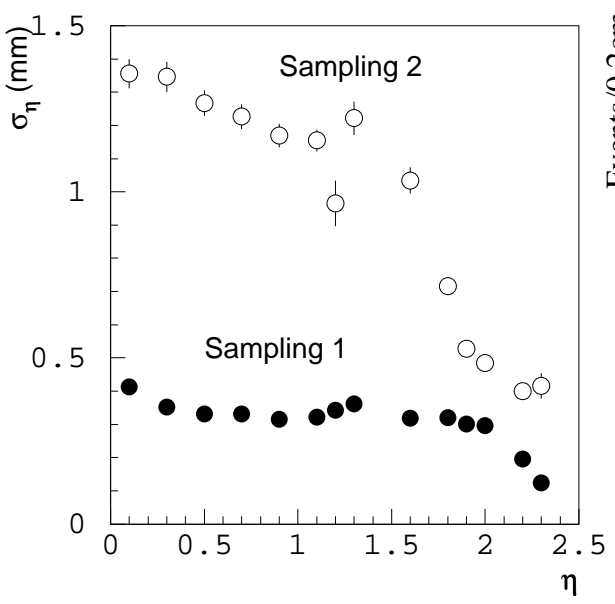

Fig. 14. Position resolution in the $\eta$ direction obtained in the first sampling (closed symbols) and in the second sampling (open symbols) of the EM Calorimeter as a function of $\eta$ for photons of $E_{T}=50 \mathrm{GeV}$.

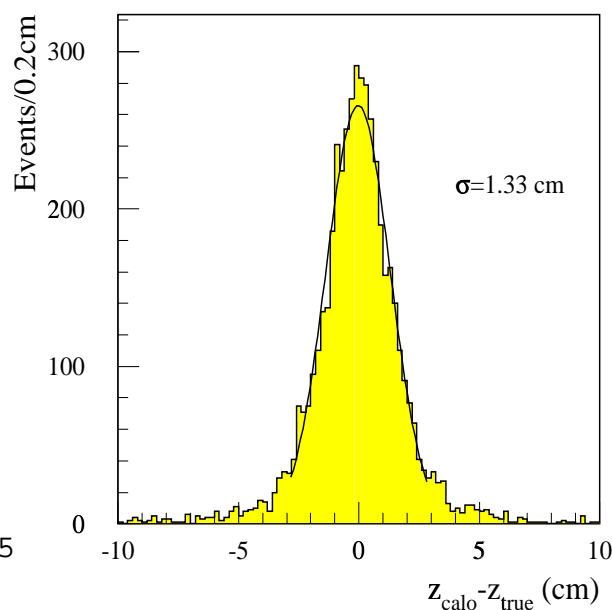

Fig. 15. Difference between the reconstructed vertex, provided by the EM Calorimeter alone and the generated vertex.

\section{Combined ID/EM Calorimeter performance}

\subsection{Photon/jet separation}

To isolate the $H \rightarrow \gamma \gamma$ signal a very good $\gamma /$ jet separation is needed. This separation is obtained using the shower shape information from the EM Calorimeter and applying the isolation cuts. The ID is used to reconstruct conversion tracks. Fig.16 shows the jet rejection after photon selection cuts as a function of the jet $E_{T}$. A rejection of better than 3000 can be obtained for $E_{T}>40 \mathrm{GeV}$, both for low and high luminosity.

The $\eta$-strips of the first calorimeter sampling also allow isolated $\pi^{0}$ and photons to be distinguished. A rejection factor of three can be achieved for a single photon efficiency of $90 \%$, which is needed in order to extract a possible $H \rightarrow \gamma \gamma$ signal over background.

The $\eta$-dependence of the $\pi^{0}$ rejection at $E_{T}=50 \mathrm{GeV}$ is shown for the low and high luminosity cases. This plot shows the $\pi^{0}$ rejection as a function of $\eta$ for low and high luminosity. For a fixed photon efficiency of $90 \%$, the electronic noise and pile-up expected at high luminosity reduce the $\pi^{0}$ rejection by less than $10 \%$.

\subsection{Electron/pion separation}

For low energy electrons it will be not easy to identify electron candidates by an unguided search in the EM Calorimeter. Instead the ID must be used to seed the calorimeter clustering. The strategy consists of several steps. First, tracks 


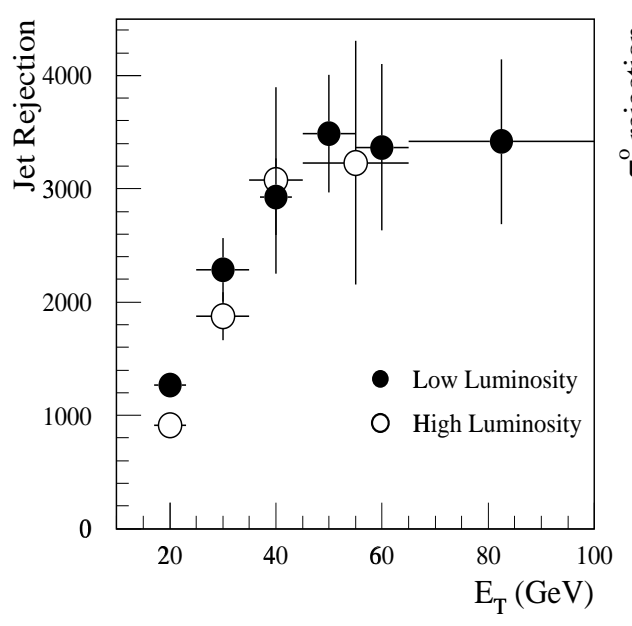

Fig. 16. Jet rejection after applying the photon selection cuts as a function of the jet $E_{T}$ for low $\left(10^{33} \mathrm{~cm}^{-2} \mathrm{~s}^{-1}\right)$ and high $\left(10^{34} \mathrm{~cm}^{-2} \mathrm{~s}^{-1}\right)$ luminosity.

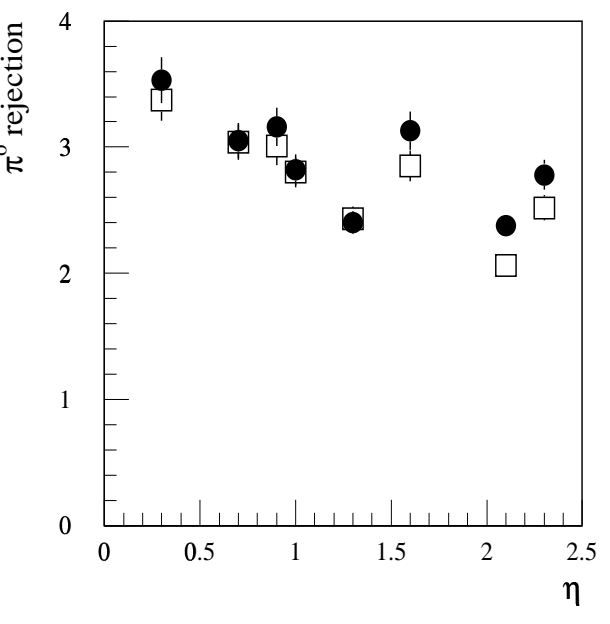

Fig. 17. Rejection of $\pi^{0}$ of $E_{T}=$ $50 \mathrm{GeV}$ for $90 \%$ photon efficiency, as a function of $\eta$, with (open squares) and without (dots) the electronic noise and the pile-up noise expected at high luminosity.

with $p_{T}>0.5 \mathrm{GeV}$ are found in the ID and then one looks in EM Calorimeter regions hit by the tracks. After that the $e-\pi$ separation can be done by cutting on a discriminating variable, based on the ID-Calo matching and on the shower shape. Fig. 18 shows the pion rejection as a function of the electron efficiency for tracks of different $p_{T}$. As one can see, this rejection depends strongly on the $p_{T}$ range examined. A rejection from 1000 to more than 10000 can be achieved for an electron efficiency between 60 and $80 \%$.

\subsection{Electron/jet separation}

The identification of isolated electrons with $p_{T}>20 \mathrm{GeV}$ will be essential for the physics searches at the LHC. A challenging task is to identify electrons in the presence of a huge QCD jets background, which is $10^{5}$ times higher, as in the case of $W$ and top decays. To separate electron from jets, cuts were developed which maintain a reasonable electron efficiency even with pile-up at high luminosity, while removing a high fraction of jet events. The cuts include special (1) Level 1, (2) Level 2 Calo, (3) Level 2 ID trigger cuts (see [9]), (4) shower shape and isolation cuts in the calorimeter, (5) cuts on track in ID, (6) cuts on ID-Calo matching in position and energy and (7) transition radiation cuts. The effect of applying all these cuts one after the other to a single electron sample and an inclusive jet sample are shown in Fig.19. The last point here corresponds to all the seven cuts applied. An electron efficiency of about $70 \%$ is retained while a jet rejection of about $1.5 \times 10^{5}$ is achieved for low luminosity and of $0.5 \times 10^{5}$ for high luminosity. It should be noted that these numbers are only low limit 


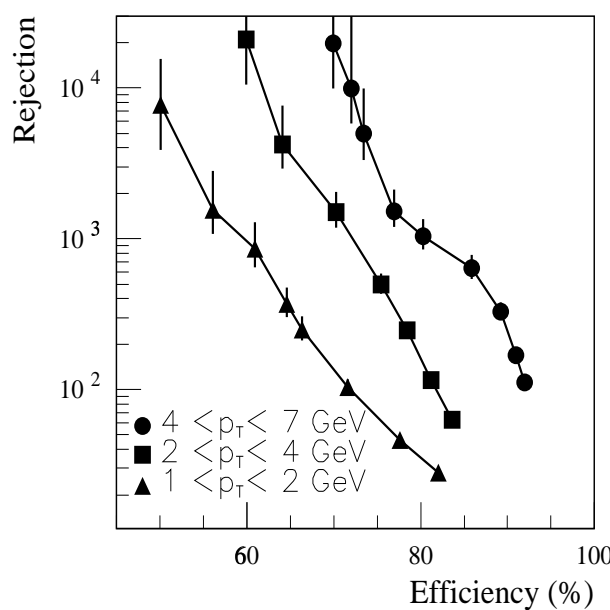

Fig. 18. Pion rejection vs electron efficiency for single particles for different $p_{T}$ ranges.

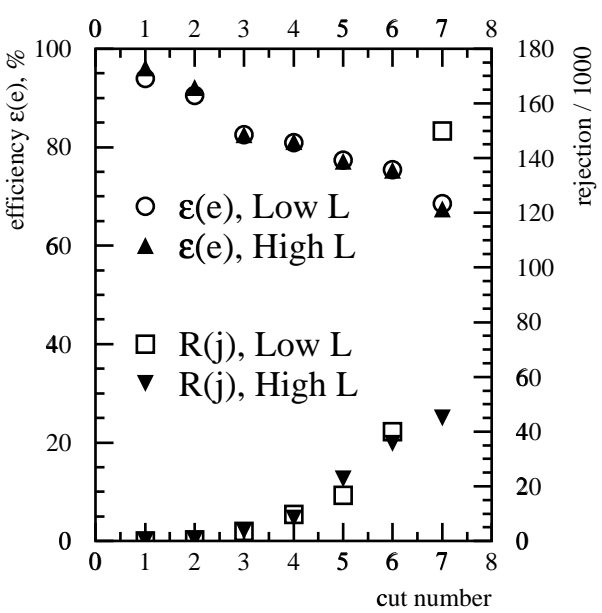

Fig. 19. Electron efficiency and jet rejection after applying subsequent cuts to an isolated electron sample and an inclusive QCD jet sample at low (open markers) and high (full markers) luminosity.

estimates, since in some cases one or even no jets from the sample were left after the cuts.

\subsection{Electron identification in the forward region}

The Forward Calorimeter in ATLAS covers the range from 3.1 to $4.9 \mathrm{in} \eta$. It has one electromagnetic and two hadronic samplings. The cells are made of tube groups parallel to the beam axis, which means a non-pointing readout structure. The transversal cell size is of the order of $1 \mathrm{~cm}$ and such a fine granularity allows to extend the electron tagging to the forward region.

The longitudinal shower profile in the Forward Calorimeter has the classical shape and electrons and pions are very well separated (Fig.20). The transversal profile is more complicated, due to the non-pointing readout structure (Fig.21). With a combination of cuts on both the longitudinal and transversal shower profiles a rejection factor of about 100 for single pions is achieved, while keeping electron efficiency at the $100 \%$ level. The effective region for tagging is $3.3<$ $|\eta|<4$.7. For electrons from $Z$ decays and dijets, a rejection factor of at least 56 can be achieved with an electron efficiency of about $50 \%$. With such a tagging it is possible to study the forward-backward asymmetry in $Z$ events and to measure the Weinberg angle with a small statistical error thanks to the fact that one electron from $Z$ decay can be identified in the forward region (see [10]). 


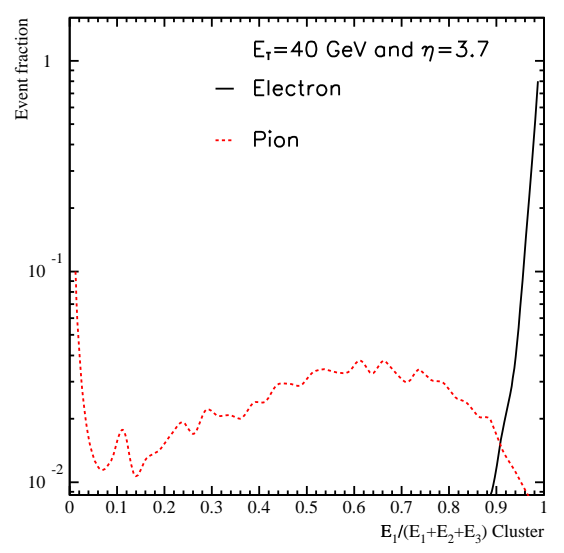

Fig. 20. Longitudinal shower profile in the Forward Calorimeter for single electrons and pions with $E_{T}=40 \mathrm{GeV}$ at $\eta=3.7$.

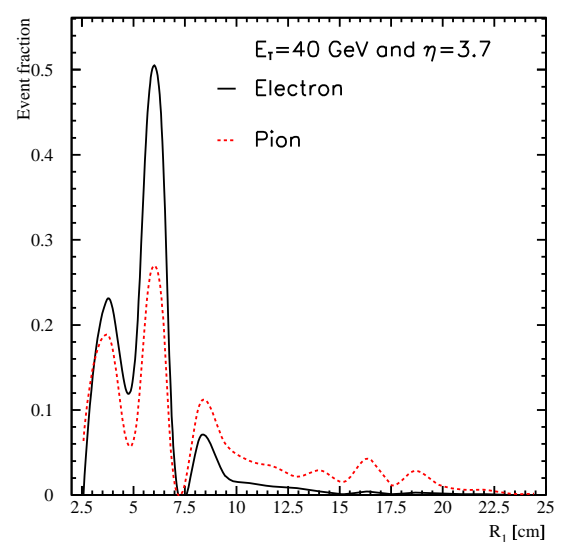

Fig. 21. Transversal shower profile in the Forward Calorimeter for single electrons and pions with $E_{T}=$ $40 \mathrm{GeV}$ at $\eta=3.7$.

\section{Muon measurements}

The conceptual design of the Muon Spectrometer is visible in Fig.22 and Fig.23. The large superconducting air-core toroid magnets are instrumented with separate trigger and high-precision tracking chambers. Over an $\eta$ range up to $|\eta|<2.7$, muon tracks are measured in three or more stations of Monitored Drift Tubes and Cathode Strip Chambers with an accuracy of better than $50 \mu \mathrm{m}$.

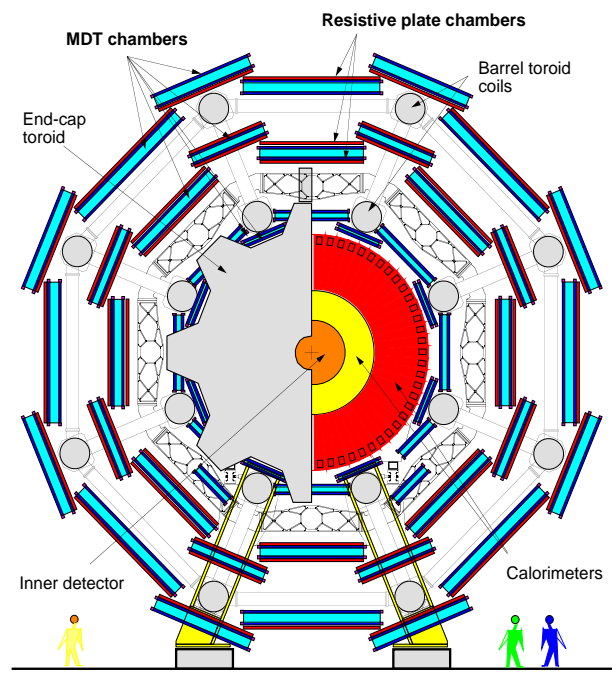

Fig. 22. Transversal view of the Muon Spectrometer.

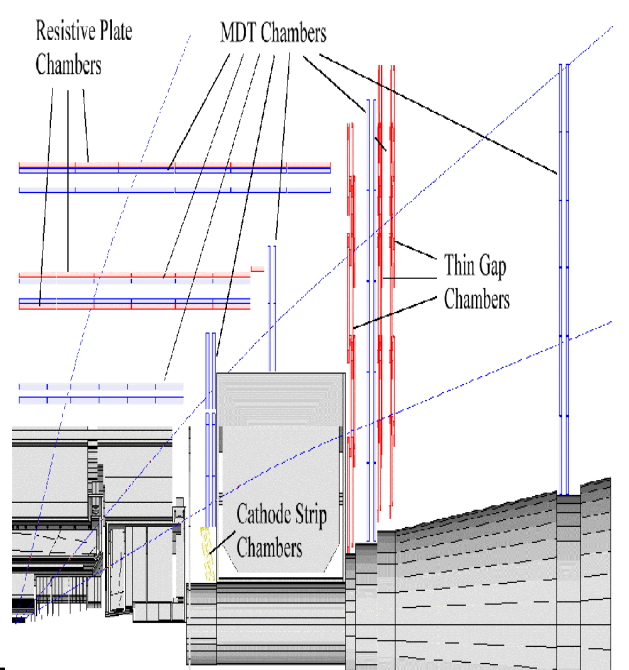

Fig. 23. Longitudinal view of the Muon Spectrometer. 


\subsection{Standalone Muon System performance}

There are various contributions to the momentum resolution given by the Muon System: the wire resolution and autocalibration, the chamber alignment, the multiple scattering and energy loss fluctuations. All these contributions are shown in Fig.24. The total resolution is about 2-3\% for muons with $p_{T}<$ $200 \mathrm{GeV}$ and it goes up to $10 \%$ for $1 \mathrm{TeV}$ particles.

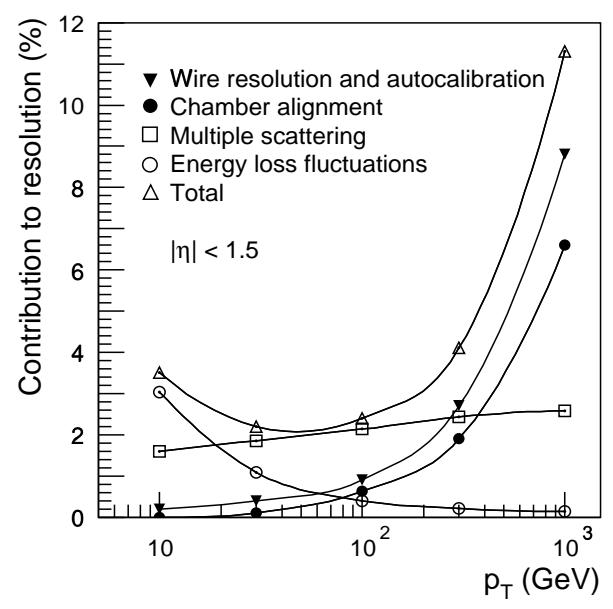

Fig. 24. Contributions to the momentum resolution of the Muon Spectrometer, averaged over $|\eta|<1.5$ and over the azimuthal angle in a standard sector.

Fig.25 and Fig.26 show the momentum resolution for $100 \mathrm{GeV}$ and $1 \mathrm{TeV}$ muons averaged over $\phi$ in one octant as a function of $\eta$. The dashed line corresponds to the bottom sector where more support structures are present. All the spikes are due to the magnet elements and to the barrel-endcap transition.

The acceptance for the muon precision chambers is computed on the basis of the so-called "superpoints" - a coincidence of at least six hits in six different sensitive planes in one station. Fig. 27 shows the acceptance for three superpoints as a function of the azimuth, integrated over $|\eta|<2.7$. The eightfold modularity of the detector is clearly seen. The drop at 270 degrees is due to a magnet feet. The Muon Spectrometer is able to reconstruct track with a slightly worse resolution using two "superpoints". The acceptance in this case is of about $99 \%$.

A track reconstruction efficiency of more than $90 \%$ can be achieved keeping the background from fake tracks at the per mil level. The single muon reconstruction efficiency and the fake rate are shown in Fig.28 as a function of the muon $p_{T}$. As the $p_{T}$ increases, more secondary particles are produced by muons, therefore increasing the fake rate. The fake track probability depends on the detector response description put in the simulation. The best results have been achieved with a fully realistic response. 

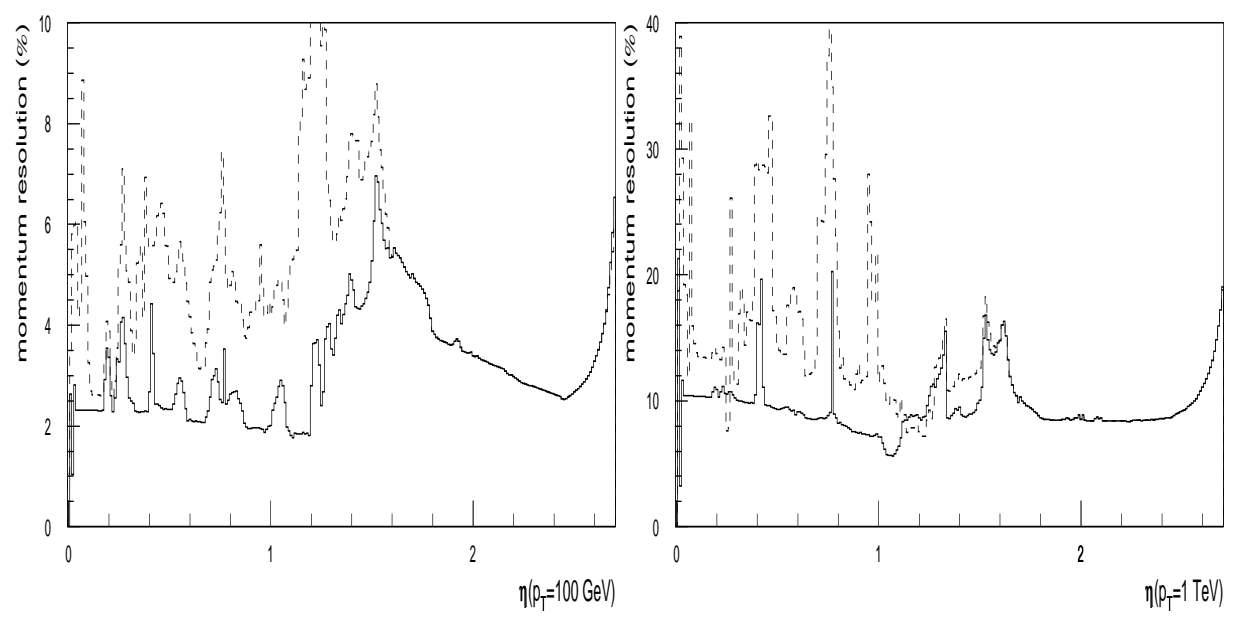

Fig. 25. Momentum resolution for $p_{T}=$ $100 \mathrm{GeV}$, averaged over $\phi$ in one octant, as a function of $\eta$. The dashed curve corresponds to the two octants that contain

Fig. 26. The same as Fig. 25 for the the feet.
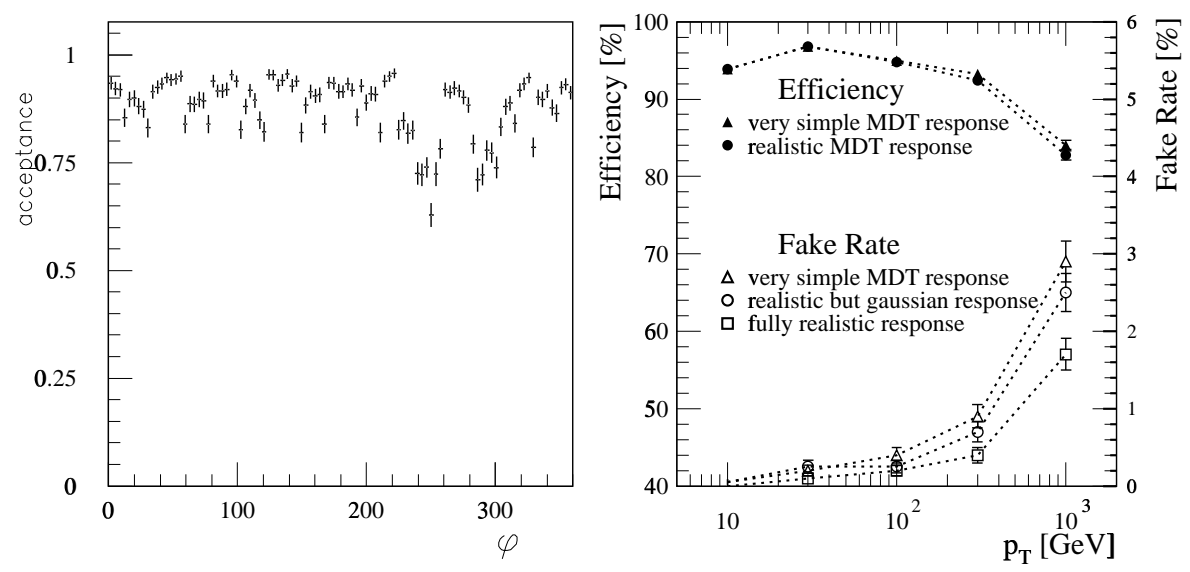

Fig. 27. Precision chamber acceptance as a function of the azimuthal angle, requiring three superpoints averaged over $\eta$.

Fig. 28. Reconstruction efficiency and fake track probability as a function of $p_{T}$ for single muons.

\subsection{Combined ID/Muon System performance}

By combining the Muon System and the ID measurements one improves the muon momentum resolution for track with $p_{T}<100 \mathrm{GeV}$, as it is shown in Fig.29. At low momentum the resolution is driven by the ID and at higher $p_{T}$ by the Muon System. The ID extends the range of precise $p_{T}$ measurements down 
to few $G e V$. Combination of the information from the ID with that from the Tile Calorimeter can lead to a good identification efficiency for muons with $p_{T}$ as low as $3 \mathrm{GeV}$.

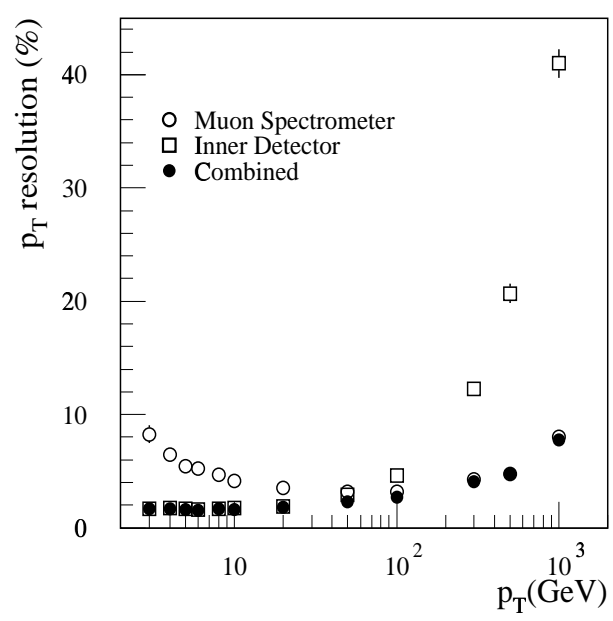

Fig. 29. $p_{T}$ resolution for tracks reconstructed in the Muon System, in the ID and for combined tracks.

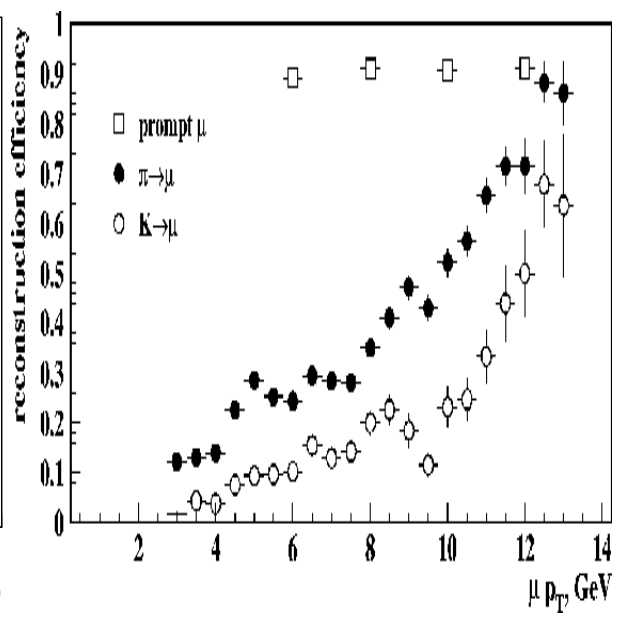

Fig. 30. Reconstruction efficiency as a function of track $p_{T}$ for prompt muons and for muons from $\pi$ and $K$ decays.

At low transverse momenta $\left(p_{T}<8 \mathrm{GeV}\right)$, in-flight decays of $\pi$ and $K$ mesons are the dominant source of muons and thus of trigger rate in the Muon System. The trigger rate can be reduced significantly by requiring a good match between tracks in the Muon System and in the ID. The reconstruction efficiency for such tracks in the Muon Spectrometer as well as the efficiency for prompt muons as a function of muon $p_{T}$ is shown in Fig.30. A rejection factor of about ten can be achieved for low- $p_{T}$ muons from $\pi / K$ decays. However, to efficiently trigger on prompt muons below $6 \mathrm{GeV}$, the information from the Tile Calorimeter is required.

\subsection{Muon tagging in the Tile Calorimeter}

The Tile Calorimeter has a total thickness of at least ten absorption lengths, and the thickness of the last longitudinal sampling is $1.9 \lambda$ in the barrel at $\eta=0$ and more than $4.5 \lambda$ in the Extended Barrel $(|\eta|>1)$. Every cell of the calorimeter is read out by two photomultipliers (PMT).

The calorimeter should be able to measure energy from few hundreds $\mathrm{MeV}$ to $T e V$ in a cell, and the muon signal coming from the last longitudinal sampling can be used for the muon Level 1 trigger.

Preliminary results from the beam test performed in the year 2001 show that the signal in the Level 1 trigger should be clearly separated from the noise (Fig.31). The evaluated muon trigger efficiency versus the purity is shown in Fig.32. It is very close to $100 \%$ for the extended barrel thanks to its thick last 
sampling. For the barrel the situation is slightly worse, but an extra factor of $\sqrt{2}$ might be achieved by summing up the signal from both PMTs in a cell.

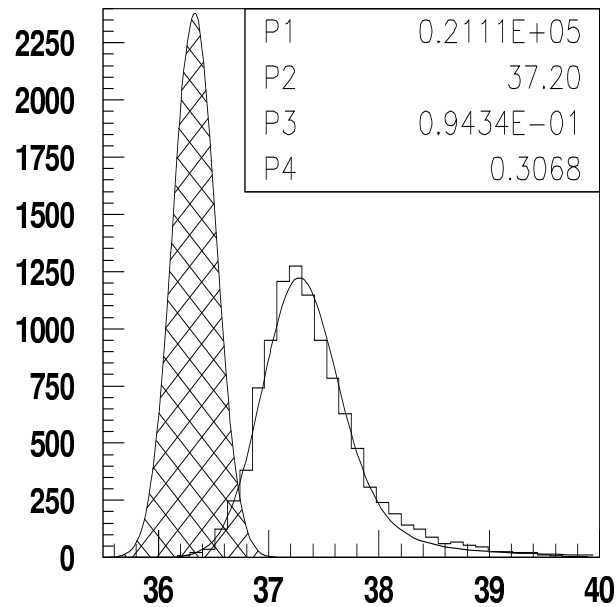

Fig. 31. Signal line shape and noise contribution in the Level 1 trigger for muons traversing the Tile Calorimeter.

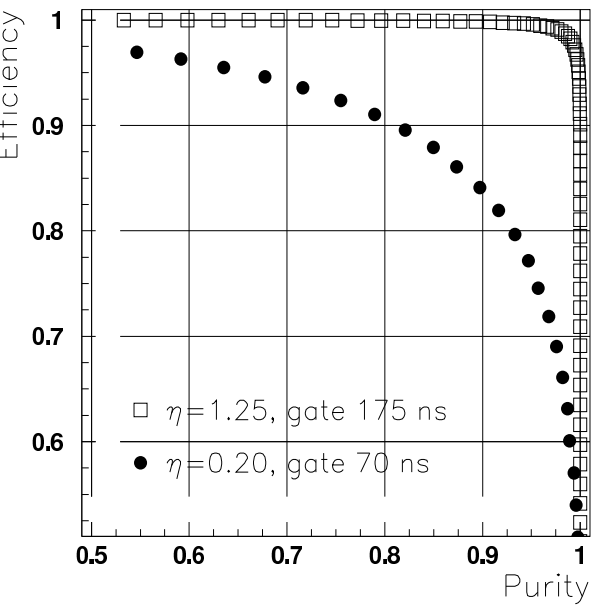

Fig. 32. Efficiency for muons in the Tile Calorimeter Barrel and Ext. Barrel as a function of the purity of the muon sample.

\section{$6 \quad$ In-situ calibration and absolute energy scale}

For the EM calibration, $Z \rightarrow e e$ decays will be mainly used, as well as $E / p$ calibration with single electrons from $W \rightarrow e \nu$ decay. The EM Calorimeter will be calibrated in 440 regions with $\Delta \eta \times \Delta \phi=0.2 \times 0.4$. Beam test measurements show that the local constant term for such a region should be of about $0.5 \%$ or less. The regions themselves can be intercalibrated with a $0.3 \%$ precision, as it is shown in Fig.33. In this plot the difference between the injected miscalibration coefficients and the coefficients measured with an "in-situ" calibration using $Z \rightarrow e e$, are shown.

Thus, summing up quadratically the $0.5 \%$ and $0.3 \%$ terms, a total constant term of less then $0.7 \%$ should be achieved. The high statistics of $Z \rightarrow e e$ allows this constant term to be achieved in less then one week of running at low luminosity. As it is shown by simulation, a $0.02 \%$ uncertainty on the EM scale is achievable. The main systematics here is the amount of material in the ID, which should be known to $1 \%$ and the errors due to bremsstrahlung.

Lower mass scales will be possibly checked using the leptonic decays of $\Upsilon$ and $J / \psi$.

For muon calibration $Z \rightarrow \mu \mu$ decays will be used. First a few runs without magnetic field will be done to calibrate the precision chambers alignment. The reconstruction of the well known $Z$ mass and the comparison of the momentum measured in the ID with the momentum in the Muon System will allow to achieve a $0.1 \%$ accuracy (see Fig.34). 


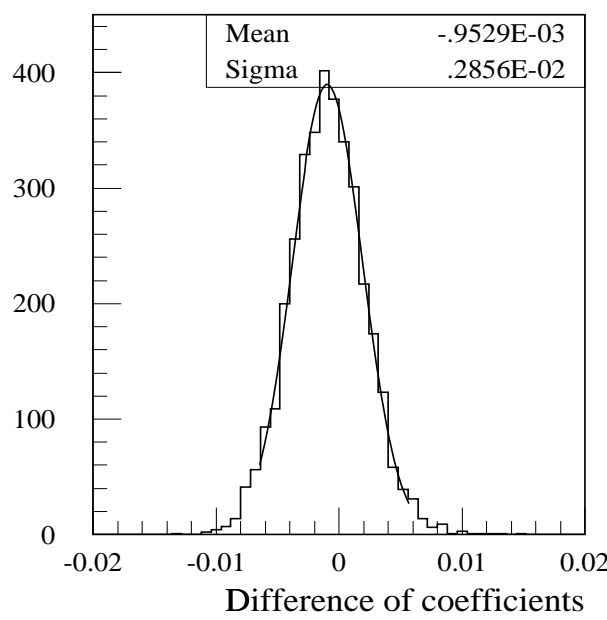

Fig. 33. Difference between the injected coefficients and the correction coefficients after the fit for the calibration of the 440 regions of the EM Calorimeter with $Z \rightarrow e e$ events.

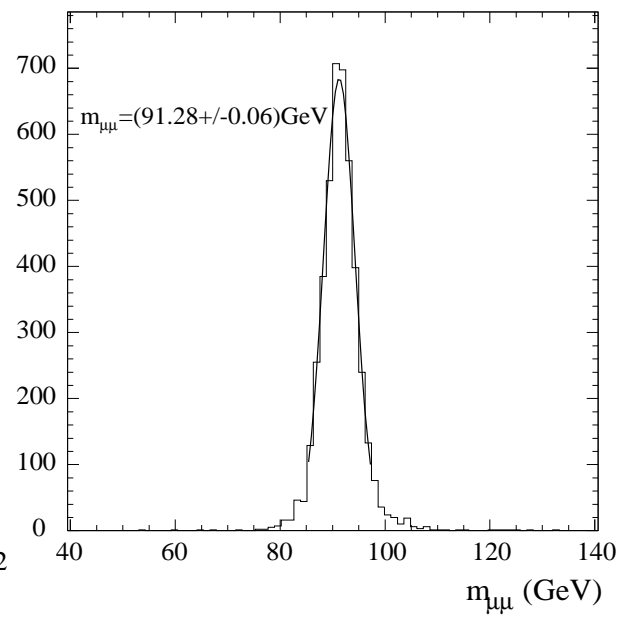

Fig. 34. Reconstructed dimuon mass for the muon calibration using $Z \rightarrow \mu \mu$.

\section{Mass reconstruction}

In Fig.35-38 one can see how the described electron, photon and muon performance of the ATLAS detector allows signal mass peak reconstruction in several physics benchmark channels. These examples show reconstructed Higgs masses in the $\gamma \gamma, 4 e, 4 \mu$ and $2 \mu$ channels. Resolution in the $\gamma \gamma$ channel is determined mostly by the EM Calorimeter performance. For the 4-leptons channels the combined Inner Detector and Calorimeter performance plays an important role, while for the case of the heavier MSSM Higgs boson which decays in $2 \mu$ the performance of the Muon Spectrometer is the major factor. Typically, the resolution is at the percent level and the acceptance in the mass bin which is used to reconstruct the signal is $80 \%$ or more, which indicates that tails are small.

\section{Conclusions}

A broad spectrum of detailed physics studies has led to the overall ATLAS detector concept, as proved by its excellent performance in identifying and measuring electrons, photons and muons.

The ATLAS Inner Detector is capable of reconstructing $\mu, \pi$ and $e$ with high efficiency and resolution. Its robustness against pile-up has been shown as well.

The EM Calorimeter is characterised by a good energy resolution, an excellent response uniformity and position/angular resolution. In addition, it provides a powerful particle identification capability, thanks to its excellent granularity.

The combination of the Inner Detector and of the EM Calorimeter, provides a very good reconstruction and identification of electrons and photons over the energy range from few $\mathrm{GeV}$ to few $\mathrm{TeV}$. 

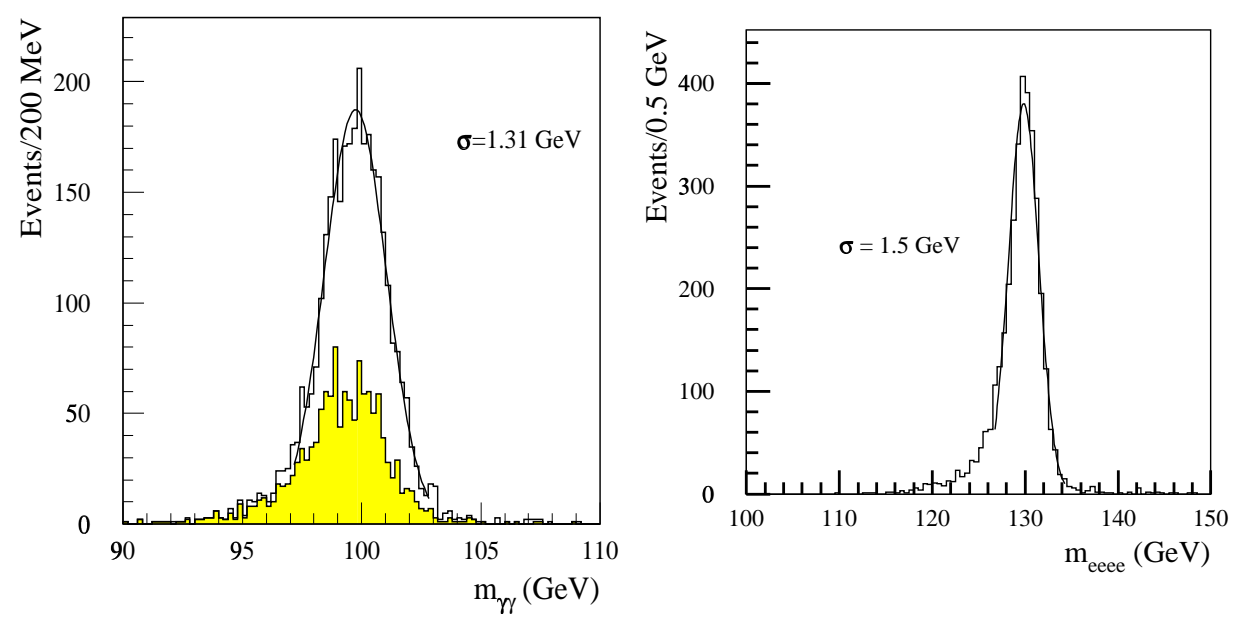

Fig. 35. $H \rightarrow \gamma \gamma$ at high luminosity, $m_{H}=100 \mathrm{GeV}$. The shaded histogram represents events containing at least one Fig. 36. $H \rightarrow Z Z^{*} \rightarrow$ eeee at low converted photon. luminosity, $m_{H}=130 \mathrm{GeV}$.

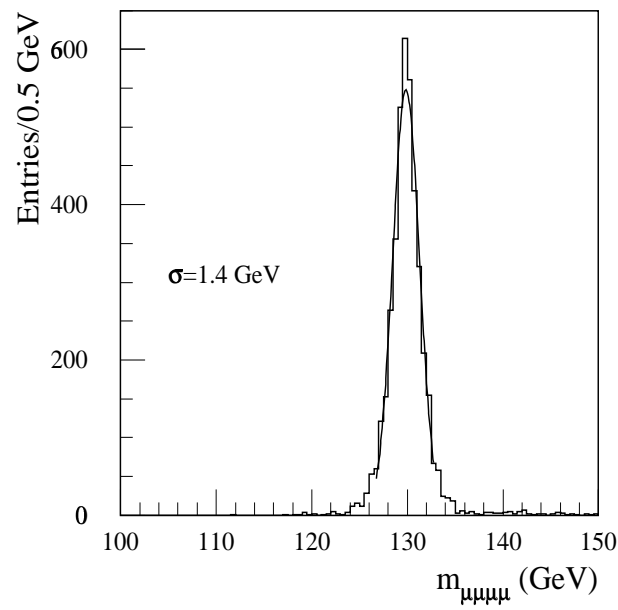

Fig. 37. $H \rightarrow Z Z^{*} \rightarrow \mu \mu \mu \mu$ at low luminosity, $m_{H}=130 \mathrm{GeV}$.

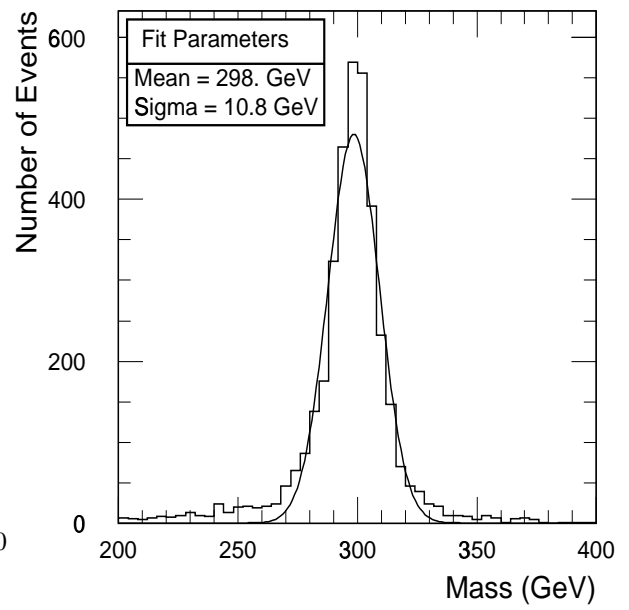

Fig. 38. $A(300) \rightarrow \mu \mu$ at low luminosity, $m_{A}=300 \mathrm{GeV}$.

With this performance, the reachable mass resolution for a light Higgs boson $\left(m_{H}=100 \mathrm{GeV}\right)$ decaying to two photons is 1.1 (1.3) $\mathrm{GeV}$ at low (high) luminosity, while the mass resolution for the four electron decays $\left(m_{H}=130 \mathrm{GeV}\right)$ is 1.5 (1.8) GeV.

Finally, the ATLAS combined muon measurements provide a robust muon identification and a high precision measurement from $6 \mathrm{GeV}$ to about $2 \mathrm{TeV}$. The accurate momentum reconstruction over such a large energy range allows a 
precise invariant mass measurement of multi-muon final states. As an example, the MSSM Higgs with $m=300 \mathrm{GeV}$ decays to two muons can be reconstructed with a mass resolution of $10 \mathrm{GeV}$.

\section{References}

1. ATLAS Collaboration, Inner Detector Technical Design Report Vol. I, CERN/LHCC/97-16 (1997)

2. ATLAS Collaboration, Inner Detector Technical Design Report Vol. II, CERN/LHCC/97-17 (1997)

3. ATLAS Collaboration, Calorimeter Performance Technical Design Report, CERN/LHCC/96-40 (1996)

4. ATLAS Collaboration, Liquid Argon Calorimeter Technical Design Report, CERN/LHCC/96-41 (1996)

5. ATLAS Collaboration, Tile Calorimeter Technical Design Report, CERN/LHCC/96-42 (1996)

6. ATLAS Collaboration, Muon Spectrometer Technical Design Report, CERN/LHCC/97-22 (1997)

7. ATLAS Collaboration, ATLAS Detector and Physics Performance Technical Design Report Vol. I, CERN/LHCC/99-14 (1999)

8. ATLAS Collaboration, ATLAS Detector and Physics Performance Technical Design Report Vol. II, CERN/LHCC/99-15 (1999)

9. S. George, ATLAS trigger and event selection, Talk at this Symposium

10. O. Rohne, Precision measurements at LHC, Talk at this Symposium 